\title{
Rationale and protocol for the Assessment of Impact of Real-time Continuous Glucose Monitoring on people presenting with severe Hypoglycaemia (AIR-CGM) study
}

\author{
Parizad Avari ${ }^{1}$, Rozana Ramli ${ }^{1}$, Monika Reddy ${ }^{1}$, Nick Oliver ${ }^{1 *}$ and Rachael Fothergill ${ }^{2}$
}

\begin{abstract}
Background: Severe hypoglycaemia carries a significant risk of morbidity and mortality for people with type 1 diabetes. Economic costs are also high, estimated at approximately $£ 13$ million annually in England, UK. Continuous glucose monitoring (CGM) has been shown to reduce hypoglycaemia and associated fear, improve overall glycaemia and quality of life, and is cost-effective. Despite effective pathways in place with high levels of resource utilization, it has been reported there are low levels of follow-up, therapy change and specialist intervention after severe hypoglycaemia. This study is designed to assess the impact of providing real-time CGM to people with type 1 diabetes, who have had a recent episode of severe hypoglycaemia (within $72 \mathrm{~h}$ ), compared to standard care.
\end{abstract}

Methods/design: Fifty-five participants with type 1 diabetes and a recent episode of severe hypoglycaemia, who are CGM naïve, will be recruited to the study. Participants will be randomised to CGM or standard care. The primary outcome is percentage time spent in hypoglycaemia $(<3.0 \mathrm{mmol} / \mathrm{L}, 55 \mathrm{mg} / \mathrm{dL})$. Secondary outcomes include other measures of hypoglycaemia, time in euglycaemia, overall glucose status and patient reported qualitative measures.

Discussion: This study assesses the impact of providing continuous glucose monitoring at the outset in individuals at highest risk of hypoglycaemia. Changing demand means that novel approaches need to be taken to healthcare provision. This study has the potential to shape future national standards.

Trial registration: NCT03748433, November 2018 (UK).

Keywords: Type 1 diabetes, Continuous glucose monitoring, Severe hypoglycaemia

\section{Background}

Intensive glucose management with insulin therapy for people with Type 1 diabetes reduces the risk of microvascular complications and cardiovascular disease [1]. However, hypoglycaemia is a common iatrogenic complication that limits individuals from safely and effectively achieving their glycaemic goals. Repeated episodes of hypoglycaemia can substantially increase the risk of severe hypoglycaemia and are associated with increased frequency and severity of moderate hypoglycaemia [2,3]

\footnotetext{
* Correspondence: nick.oliver@imperial.ac.uk

'Division of Diabetes, Endocrinology and Metabolism, Faculty of Medicine, Imperial College, 757a, Commonwealth Building, Hammersmith Campus, Du Cane Road, W12 OHS, London, UK

Full list of author information is available at the end of the article
}

and impaired awareness of hypoglycaemia [4]. Moreover, a preceding episode of severe hypoglycaemia is a powerful predictor of subsequent episodes of hypoglycaemia, independent of treatment intensity [5].

In adults, severe hypoglycaemia is defined as any episode of hypoglycaemia requiring the assistance of a third party to actively administer carbohydrate, glucagon, or take other corrective actions. On average, people with Type 1 diabetes report 1.8 self-treated incidences of hypoglycaemia per week, and $0.2-3.2$ episodes of severe hypoglycaemia annually $[6,7]$. This may be an underestimate.

Severe hypoglycaemia is associated with significant morbidity and even mortality, provoking major vascular events and causing neurological disability [1]. Between 4 and $10 \%$ of deaths in people with type 1 diabetes are

(c) The Author(s). 2019 Open Access This article is distributed under the terms of the Creative Commons Attribution 4.0 International License (http://creativecommons.org/licenses/by/4.0/), which permits unrestricted use, distribution, and reproduction in any medium, provided you give appropriate credit to the original author(s) and the source, provide a link to the Creative Commons license, and indicate if changes were made. The Creative Commons Public Domain Dedication waiver (http://creativecommons.org/publicdomain/zero/1.0/) applies to the data made available in this article, unless otherwise stated. 
attributed to hypoglycaemia [8] and the risk of severe hypoglycaemia increases 6-fold in people with impaired awareness of hypoglycaemia [9].

The impact of hypoglycaemia on health systems is widespread and includes both acute and chronic complications. In the UK diabetes consumes more than $10 \%$ of the National Health Service (NHS) budget [10] and in the USA a relatively greater amount is spent on type 1 compared with type 2 diabetes $(8.6 \%$ of the diabetes budget compared with $5.6 \%$ of diabetes prevalence) [11].

The mean costs per hospital admission for hypoglycaemia in England is estimated to be in excess of $£ 1000$, with a total direct cost of severe hypoglycaemic episodes of around £13million each year [12-14]. Although hospital admissions for hypoglycaemia represent a small proportion of emergency department visits, they have substantial resource implications [15].

To address hypoglycaemia risk, regular self-monitoring of blood glucose up to 4-10 times daily and structured education, such as DAFNE (Dose Adjustment for Normal Eating) programme, are advocated in the National Institute of Clinical Excellence (NICE) guidance [16]. Despite such developments, severe hypoglycaemia remains a major hazard.

Continuous glucose monitoring (CGM) devices display an estimate of blood glucose levels, with alerts and alarms for impending and established hypo- and hyperglycaemia. In type 1 diabetes, real-time CGM has been shown to reduce hypoglycaemia $[12,17,18]$, and improve overall glycaemia in all age groups when used continuously. Furthermore, CGM is associated with improvements in quality of life [19], reduced hypoglycaemia fear [20], and is cost-effective [21].

In the UK, CGM is supported by NICE for people with type 1 diabetes who are willing to commit to using CGM at least $70 \%$ of the time and who have any of the following despite optimised use of insulin therapy and conventional blood glucose monitoring [22]:

- More than 1 episode a year of severe hypoglycaemia with no obvious preventable precipitating cause.

- Complete loss of awareness of hypoglycaemia.

- Frequent (more than 2 episodes a week) asymptomatic hypoglycaemia that is causing problems with daily activities.

- Extreme fear of hypoglycaemia.

- Hyperglycaemia (HbA1c level of $75 \mathrm{mmol} / \mathrm{mol}$ [9\%] or higher) that persists despite testing at least 10 times a day.

Over the last two decades, with growing demands on the NHS, emergency medical and ambulance services have been required to redefine their role and configuration. Emergency departments are under increased pressure to discharge individuals from the emergency department directly, with review by specialist diabetes nurses or outpatient follow-up with the diabetes team.

Similar to the rest of the UK, the London Ambulance Service NHS Trust (LAS) have developed integrated care pathways for hypoglycaemia management [23]. The referral pathway enables people with known diabetes requiring assistance for a hypoglycaemic episode to be assessed and treated by LAS, before either being transferred to hospital, or referred to their general practitioner or local NHS specialist diabetes service.

Despite development of effective hypoglycaemia pathways within emergency departments and ambulance services to primary, community and secondary care services for early review [24-26], it has been reported that there are low levels of follow-up, therapy change and specialist input following an episode of hypoglycaemia [27]. This is in line with the clinical experience of the authors.

The aims of this study "Assessment of the Impact of Real-time Continuous Glucose Monitoring on People Presenting with Severe Hypoglycaemia (AIR-CGM)" is to assess the impact of real-time CGM in people with type 1 diabetes, who have had a recent episode of severe hypoglycaemia (within $72 \mathrm{~h}$ ), compared to usual care. The International Hypoglycaemia Study Group recommend reporting hypoglycaemia in studies as $<3.0 \mathrm{mmol} /$ 1 , and is therefore used as the basis for the primary study outcome [28].

This is a collaborative study with the London Ambulance Service and to our best knowledge the first study to evaluate the impact of initiating real-time CGM soon after an episode of severe hypoglycaemia requiring an ambulance call-out or admission to the Emergency Department.

\section{Methods/Design \\ Design}

This is a randomised, controlled trial comparing the impact of real-time CGM with usual care following severe hypoglycaemia (Fig. 1). Fifty-five participants will be followed up for 12 weeks.

\section{Eligibility - inclusion criteria}

Participants are included if:

- Adults aged > 18 years;

- Episode of severe hypoglycaemia requiring ambulance call-out or emergency department attendance within $72 \mathrm{~h}$;

- Type 1 diabetes confirmed on the basis of clinical features;

- Type 1 diabetes for greater than 3 years 


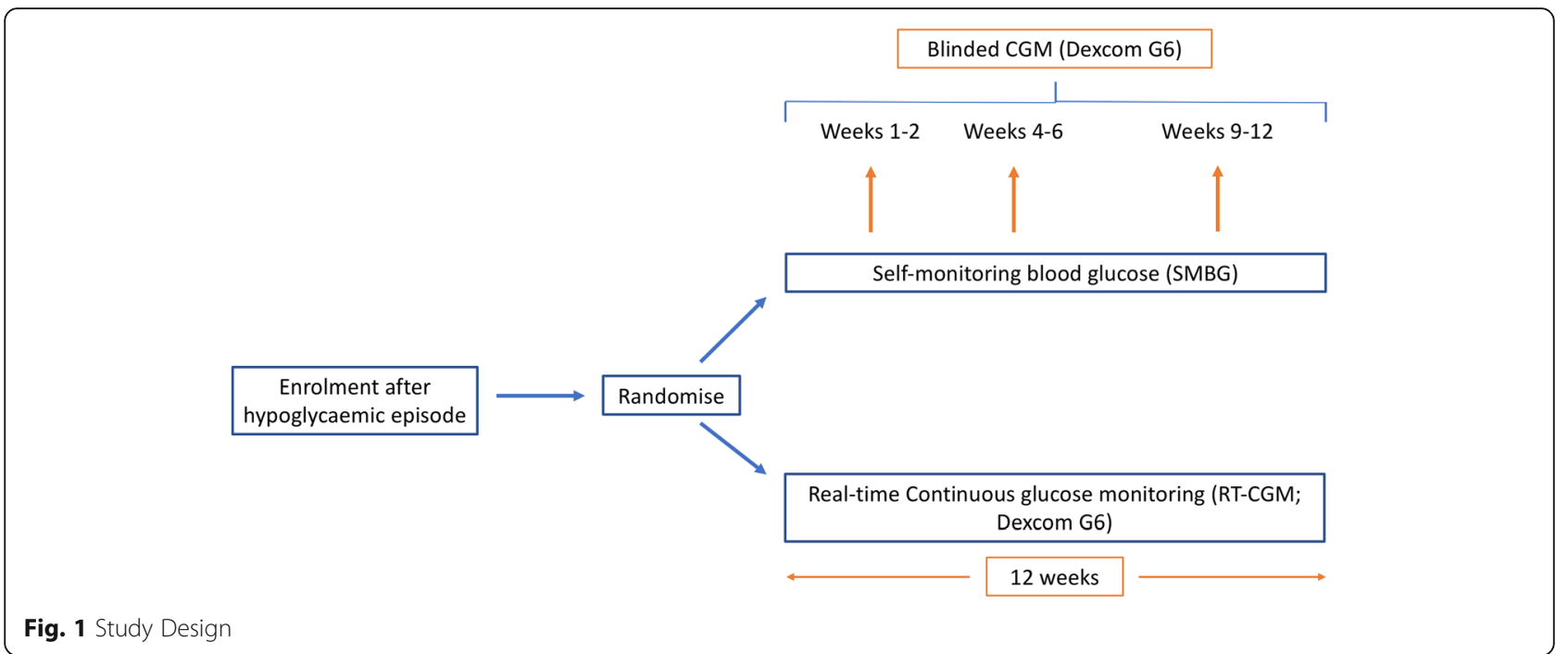

Eligibility - exclusion criteria

Participants are excluded if:

- CGM or flash glucose monitoring (Abbott Freestyle Libre device) used within the last 6 months (except for short periods of diagnostic blinded use under clinic supervision)

- Use of pre-mixed insulin;

- Pregnant or planning pregnancy;

- Breastfeeding;

- Enrolled in other clinical trials;

- Active malignancy or under investigation for malignancy;

- Reduced manual dexterity;

- Severe visual impairment;

- No access to smartphone or computer;

- Unable to participate due to other factors, as assessed by the Chief Investigators.

The aim of this study is to recruit people with type 1 diabetes at high-risk for recurrent severe hypoglycaemia who may benefit from CGM usage. The study population is in keeping with other clinical studies of adults at highest risk of hypoglycaemia (eg. HypoDE [29], IN CONTROL [30] and I-HART CGM [31]) and will include participants with long diabetes duration, hypoglycaemia-associated autonomic failure, as well as people experiencing their first episode of severe hypoglycaemia resulting from transient risk factors. People with enduring risks that may not be modified by CGM (such as drug or alcohol use) will be assessed on an individual basis and may be excluded with these data reported at the study end.

\section{Sample size and feasibility}

London Ambulance Service NHS Trust (LAS) provides emergency services to a population of 8.6 million in
London. The Ambulance service covers an area of approximately 620 mile $^{2}$, supporting 39 Acute Trusts and 32 primary care Clinical Commissioning Groups (CCG). LAS attends approximately 20,000 diabetes related emergencies per year [23]. In 2015, the LAS recorded 2152 emergency calls for hypoglycaemia within North West London only (i.e. Harrow, Hillingdon, Brent, North Central, Hounslow and Hammersmith/Fulham). Of these, $64 \%$ of callouts resulted in conveyance to the Emergency Department. Furthermore, between 1st of April 2017 and 31st of March 2018 the LAS received 10,490 emergency calls for hypoglycaemia across Greater London, with $58.78 \%$ of these incidents conveyed to Emergency Departments.

In terms of attendances to the emergency departments, between 1st March 2014 and 28th February 2015, there were 236 attendance episodes at Imperial College Healthcare NHS Trust, with a coded diagnosis of hypoglycaemia.

Based on our previous findings from technology trials, we estimate the percentage time spent in hypoglycaemia $(<3.0 \mathrm{mmol} / \mathrm{l})$ to be $64 \%$ lower in the CGM group compared to the self-monitoring group [mean (SD): CGM group 2.1\% (2.3\%); self-monitoring group 5.8\% (5.9\%)]. To demonstrate that difference as significant at $p<0.05$ and with $80 \%$ power, 25 participants would be needed in each group. To allow for a $10 \%$ drop-out 55 participants will be recruited.

\section{Recruitment}

Recruitment is undertaken in collaboration with the LAS and the Emergency Departments of local NHS hospitals serving a diverse population in London (St Mary's Hospital and Charing Cross Hospital; Fig. 2). LAS's Referral Support Team will undertake pre-screening eligibility checks of individuals who have experienced severe hypoglycaemia and recover well enough following treatment by LAS clinicians 


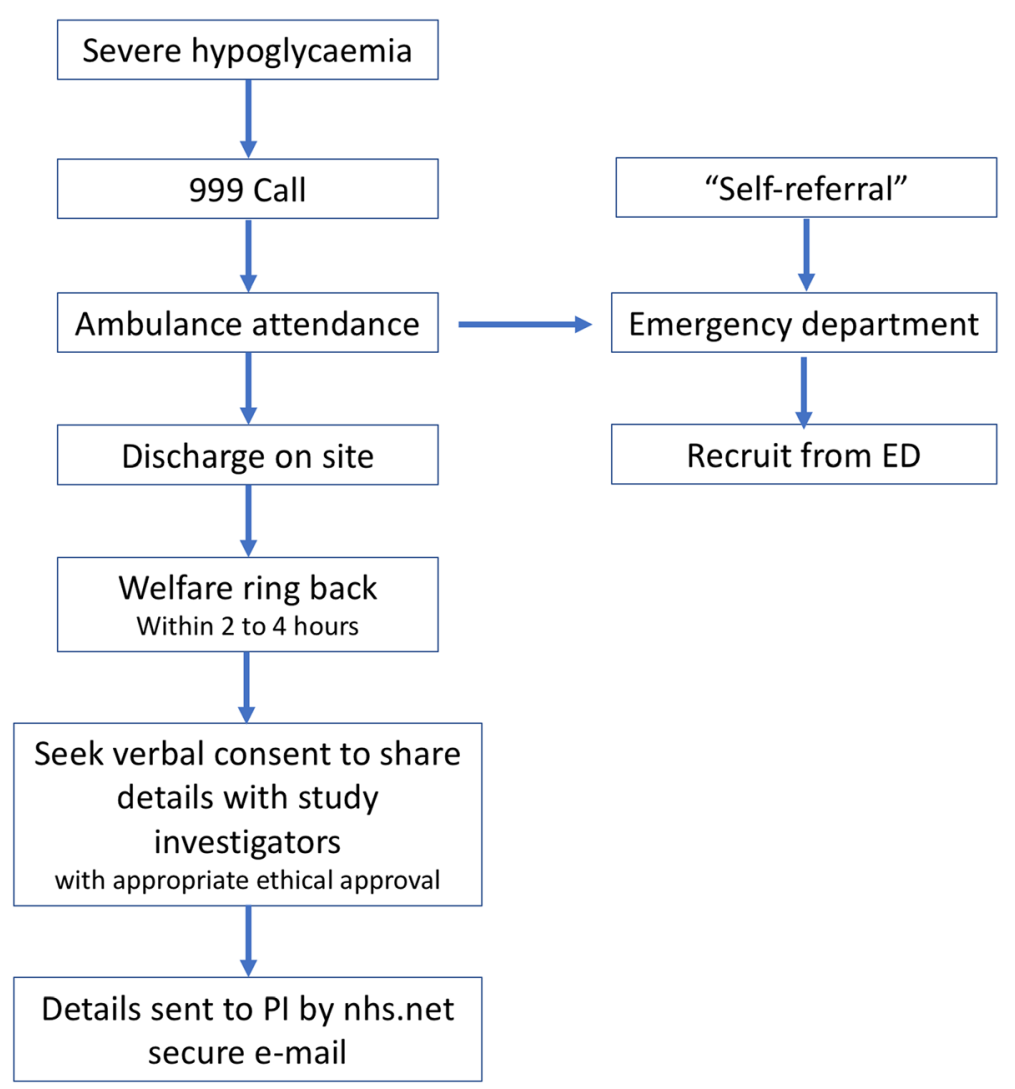

Fig. 2 Recruitment Flow Chart

to be left on scene (not transported to hospital). If the patient meets the study inclusion criteria, their details will be passed to a clinician in the Emergency Operations Centre who will contact the person within $2 \mathrm{~h}$ by telephone to check on their welfare. If the person has capacity and is well, the clinician will introduce the study and gain verbal consent to pass their name and telephone number, securely, to the research team (Fig. 2).

Potential participants will be identified from the Emergency Departments on a daily retrospective database search on admissions using the diagnosis code "hypoglycaemia".

\section{Screening visit and enrolment}

Following informed consent, study participants will give a full medical and medication history, as well as undergo a physical examination and ECG. Venous blood will be taken at 9 am for measurement of HbA1c, plasma glucose, renal function, serum C-peptide, thyroid function test, 9 am cortisol and coeliac screen (tissue transglutaminase antibody). A random urine sample will also taken for measurement of albumin:creatinine ratio, and women of childbearing age will have a urine pregnancy test to exclude pregnancy. If participants meet the inclusion criteria, they will be enrolled on to the study and complete questionnaires (Diabetes treatment satisfaction questionnaire
[DTSQ], Gold Score, CGM usability, Problem Areas in Diabetes [PAID], and Fear of hypoglycaemia survey score [HFS-2]). Potential participants will be excluded if adrenal insufficiency is identified and will undergo appropriate investigation and treatment to address this. Additionally, if thyroid function is overtly abnormal or untreated coeliac disease is identified, as this may contribute to their ongoing potential for hypoglycaemia, the participant will be withdrawn.

Enrolled participants will be randomised to real-time CGM or self-monitoring blood glucose (SMBG) group. Randomisation to be performed using sealedenvelope. com and stratified by insulin delivery modality.

The real-time CGM group will receive Dexcom G6 transmitter and sensors, as well as a structured education refresher focusing on hypoglycaemia avoidance, recognition, and management. Participants in the SMBG group will receive the same structured education refresher focusing on hypoglycaemia avoidance, recognition, and management. All education is delivered from a predefined curriculum and is supported by independent written materials.

Participants will be instructed to test their capillary blood glucose if symptoms of hypo- or hyperglycaemia occur, in case of sensor failure or if the sensor glucose is 
out of the desired range $(3.9 \mathrm{mmol} / \mathrm{L}-13.3 \mathrm{mmol} / \mathrm{L}, 70$ $\mathrm{mg} / \mathrm{dL}-240 \mathrm{mg} / \mathrm{dL}$ ). Sensor change for the Dexcom G6 is required every 10 days as per manufacturer's guidance (or sooner in event of sensor failure). Low glucose alert settings are standardised at $4.4 \mathrm{mmol} / \mathrm{L}(80 \mathrm{mg} / \mathrm{dL})$ for all participants at the start of the study and may be reduced to $4.0 \mathrm{mmol} / \mathrm{L}(70 \mathrm{mg} / \mathrm{dL})$ at week 2 during the telephone visit depending on participant preference. High glucose alerts can be personalised.

The SMBG group will additionally undergo blinded CGM at weeks 1 and 2, weeks 4 to 6 and weeks 9 to 12 using the Dexcom G6 system. Participants in this group will be shown how to insert the Dexcom G6 at the first clinic visit and sensors are provided so they can do this at home.

\section{Follow-up visits}

Participants will be provided telephone support twice in the first week, then weekly for the next 3 weeks, and every 2 weeks thereafter, to optimise glucose and reduce the risk of further hypoglycaemia.

Participants in the CGM group with an iOS or Android smartphone will be able to upload and share data with the Research team through the manufacturer's software. Participants who do not have a smartphone can upload their CGM data using a PC or Mac desktop computer with their CGM receivers. In total, participants will use CGM for 12 weeks.
Participants in the SMBG group will be asked to selfmonitor a minimum of 4 times daily and upload data using appropriate software. They will receive telephone support from the research team, based on their SMBG data, at the same time points as the CGM group, to optimise glucose and reduce the risk of further hypoglycaemia. Blinded CGM data from the SMBG group will be uploaded at the end of the study.

\section{End of study}

At the end of the study (i.e. at end of 12 weeks), repeat blood tests to assess HbA1c will be performed and questionnaires similar to that at baseline will be completed. Study equipment is returned, and individuals will resume to their standard care. Table 1 outlines a summary of participant visits.

\section{Study Outcomes \\ Primary outcome}

The primary outcome is percentage time spent in hypoglycaemia $(<3.0 \mathrm{mmol} / \mathrm{L}, 55 \mathrm{mg} / \mathrm{dL})$.

\section{Secondary outcomes}

- Number of episode of serious hypoglycaemia (defined as a sensor glucose $<3.0 \mathrm{mmol} / \mathrm{L}(55 \mathrm{mg} / \mathrm{dL})$ for $>20 \mathrm{~min}$ )

Table 1 Summary table of investigations, treatment and assessments

\begin{tabular}{|c|c|c|c|c|c|c|c|c|c|c|c|c|c|}
\hline & \multirow{2}{*}{$\begin{array}{c}\text { Clinic Visit } 1 \\
\text { (Screening \& } \\
\text { Randomisation) }\end{array}$} & \multicolumn{12}{|c|}{ End of Week } \\
\hline & & 1 & 2 & 3 & 4 & 5 & 6 & 7 & 8 & 9 & 10 & 11 & $\begin{array}{c}\text { Week } 12 \text { (Clinic } \\
\text { Visit 2) }\end{array}$ \\
\hline History & $x$ & & & & & & & & & & & & \\
\hline Medication history & $x$ & & & & & & & & & & & & \\
\hline ECG & $\mathrm{x}$ & & & & & & & & & & & & \\
\hline Physical examination & $x$ & & & & & & & & & & & & \\
\hline Urine pregnancy test & $\mathrm{x}$ & & & & & & & & & & & & \\
\hline $\mathrm{HbA} 1 \mathrm{c}$ & $x$ & & & & & & & & & & & & $\mathrm{x}$ \\
\hline Glucose & $x$ & & & & & & & & & & & & \\
\hline Renal function & $\mathrm{x}$ & & & & & & & & & & & & \\
\hline TFT & $x$ & & & & & & & & & & & & \\
\hline 9am cortisol & $x$ & & & & & & & & & & & & \\
\hline Coeliac screen & $x$ & & & & & & & & & & & & \\
\hline C-peptide & $\mathrm{x}$ & & & & & & & & & & & & \\
\hline Gold Score & $\mathrm{x}$ & & & & & & & & & & & & $\mathrm{x}$ \\
\hline Participant interview & $\mathrm{x}$ & & & & & & & & & & & & $\mathrm{x}$ \\
\hline QoL Questionnaires & $x$ & & & & & & & & & & & & $x$ \\
\hline Diabetes Education & $\mathrm{x}$ & & & & & & & & & & & & \\
\hline Randomisation & $\mathrm{x}$ & & & & & & & & & & & & \\
\hline CGM education & $\mathrm{x}$ & & & & & & & & & & & & \\
\hline CGM insertion & $\mathrm{x}$ & $\mathrm{x}$ & $\mathrm{x}$ & $\mathrm{x}$ & $\mathrm{x}$ & $\mathrm{x}$ & $\mathrm{x}$ & $\mathrm{x}$ & $\mathrm{x}$ & $\mathrm{x}$ & $\mathrm{x}$ & $\mathrm{x}$ & \\
\hline Telephone consultation & & $x$ (twice) & $x$ & $x$ & $x$ & & $x$ & & $x$ & & $x$ & & . \\
\hline $\begin{array}{c}\text { CGM group - CGM } \\
\text { download }\end{array}$ & & x (twice) & $\mathrm{x}$ & $\mathrm{x}$ & $x$ & & $\mathrm{x}$ & & $x$ & & $\mathrm{x}$ & & $x$ \\
\hline $\begin{array}{c}\text { SMBG Group -Meter } \\
\text { download }\end{array}$ & & $x$ (twice) & $x$ & $\mathrm{x}$ & $\mathrm{x}$ & & $x$ & & $x$ & & $\mathrm{x}$ & & $x$ \\
\hline $\begin{array}{l}\text { SMBG Group - Blinded } \\
\text { CGM download }\end{array}$ & & & $x$ & & & & $x$ & & & & & & $x$ \\
\hline Research clinic visit & $\mathrm{x}$ & & & & & & & & & & & & $x$ \\
\hline
\end{tabular}


- Number of episodes of severe hypoglycaemia requiring third party assistance

- Percentage time spent in hypoglycaemia (<3.9 mmol/L, $70 \mathrm{mg} / \mathrm{dL})$

- Percentage time in euglycaemia $(3.9-7.8 \mathrm{mmol} / \mathrm{L}$, $70-140 \mathrm{mg} / \mathrm{dL}$ )

- Percentage time spent in target $(3.9-10 \mathrm{mmol} / \mathrm{L}$, $70-180 \mathrm{mg} / \mathrm{dL}$ )

- Percentage time spent in hyperglycaemia (> $10 \mathrm{mmol} / \mathrm{L}, 180 \mathrm{mg} / \mathrm{dL}$ )

- Number hypoglycaemic excursions

- Glucose variability

- HbAlc

- Ambulance call-out rates

- Summary measure of quality of life from questionnaires (DTSQ, CGM usability, PAID, HFS2)

- Gold score

- Cost effectiveness

\section{Ethics, informed consent and safety}

The study is conducted in accordance with the recommendations for physicians involved in research on human subjects adopted by the 18th World Medical Assembly, Helsinki 1964 and later revisions.

Written consent is obtained from each participant by the research team after full explanation, an information leaflet offered, and time allowed for consideration. The right of the participant to refuse to participate, or continue participating, without giving reasons and without prejudicing further treatment is respected. The Chief Investigator ensures that participant confidentiality is respected, and local data protection requirements are met, in line with the Data Protection Act 2018.

Only research staff trained in Good Clinical Practice participate in the project to obtain informed consent and conduct procedures. The study is subject to inspection and audit by the sponsors, ensuring adherence to Good Clinical Practice and other aspects of research governance.

All adverse events (serious and non-serious) will be reported to the Chief Investigator in the first instance.

\section{Data}

Data are locally collected and transferred to the central database. The data are coded and anonymised and then stored in an encrypted folder in a password protected computer.

\section{Publication policy}

The results arising from this project will be disseminated by peer reviewed scientific journals, internal report, conference presentation and publication on websites. No identifiable personal data will be published.

All participants will be informed of the results at the conclusion of the study and details of any publications that arise from the study will be disseminated to participants.

\section{Statistics and data analysis}

Analyses will be performed using Stata version 14.2. Baseline data will be taken from the first 14 days of monitoring and outcomes will be calculated from the last 30 days of the treatment period.

Analysis is based on an intention to treat principle. The primary and secondary outcomes will be assessed between the two study arms using a Wilcoxon Rank sum test. Other outcomes assessed will include hypoglycaemia awareness by participant (Gold score). Hypoglycaemia outcomes will be assessed throughout the time period and nocturnally (22:00-06:00 h).

\section{Discussion}

This study is the first to evaluate the impact of real-time CGM initiation, compared to usual care, following an episode of severe hypoglycaemia requiring an ambulance call-out or admission to the Emergency Department.

Addressing severe hypoglycaemia to reduce the risk of further episodes and acting promptly to optimise hypoglycaemia awareness is critical in people at high risk. If the results of this study are positive, enabling individuals to have direct access to CGM in a timeeffective manner following severe hypoglycaemia, may provide an effective adjunct to diabetes self-management with improved glycaemia, greater treatment satisfaction and associated reduction in costs to the National Health Service and Ambulance services.

\section{Abbreviations}

CCG: Clinical Commissioning Group; CGM : Continuous Glucose Monitoring; DAFNE: Dose Adjustment for Normal Eating; DTSQ: Diabetes treatment satisfaction questionnaire; HFS-2: Fear of hypoglycaemia survey score; LAS: London Ambulance Service; NHS: National Health Service; NICE: National Institute of Clinical Excellence; PAID: Problem Areas in Diabetes; SD: Standard deviation; SMBG: Self-monitoring blood glucose; UK: United Kingdom; USA: United States of America

\section{Acknowledgements}

We would like to thank the NIHR Imperial Biomedical Research Centre and the NIHR Imperial Clinical Research Facility for infrastructure support. The views expressed are those of the author(s) and not necessarily those of the $\mathrm{NHS}$, the NIHR or the Department of Health and Social Care.

\section{Author contributions}

NO, MR and RF designed the study. PA and RR wrote the first draft of the manuscript. All authors have read and approved the final manuscript.

\section{Funding}

Dexcom funded this investigator-initiated study and will provide materials. The funding body had no role in the collection, analysis, and interpretation of data; and in writing the manuscript.

Availability of data and materials Not applicable 


\section{Ethics approval and consent to participate}

This study has been given ethical approval for conduct in the NHS by the Health Research Authority - London Bromley Research Ethics Committee (reference 18/LO/1525). The sponsor for the study is Joint Research Compliance Office, Imperial College London. Written informed consent to participate in the study was obtained from all participants.

\section{Consent for publication}

Not applicable

\section{Competing interests}

NO has received research funding from Roche diabetes and Dexcom, and has participated in advisory groups for Dexcom, Medtronic and Roche Diabetes. MR has received research funding from Dexcom, and has participated in advisory groups for Dexcom and Roche Diabetes. PA, RR and RF have no conflict of interests.

\section{Author details}

'Division of Diabetes, Endocrinology and Metabolism, Faculty of Medicine, Imperial College, 757a, Commonwealth Building, Hammersmith Campus, Du Cane Road, W12 OHS, London, UK. ${ }^{2}$ Clinical Audit \& Research Unit, London Ambulance Service NHS Trust, London, UK.

Received: 21 February 2019 Accepted: 9 October 2019

\section{Published online: 26 October 2019}

\section{References}

1. Nathan DM, Cleary PA, Backlund JY, Genuth SM, Lachin JM, Orchard TJ, et al. DCCT trial intensive diabetes treatment and cardiovascular disease in patients with type 1 diabetes. N Engl J Med. 2005;353(25):2643-53.

2. Cryer PE. Hypoglycaemia: the limiting factor in the glycaemic management of type I and type II diabetes. Diabetologia. 2002;45(7):937-48.

3. The Diabetes Control and Complications Trial Research Group. Hypoglycemia in the Diabetes Control and Complications Trial. Diabetes. 1997:46(2):271-86.

4. Graveling AJ, Frier BM. Impaired awareness of hypoglycaemia: a review. Diabetes Metab. 2010;36(Suppl 3):S64-74.

5. Gubitosi-Klug RA, Braffett BH, White NH, Sherwin RS, Service FJ, Lachin JM, et al. Risk of severe hypoglycemia in type 1 diabetes over 30 years of follow-up in the DCCT/EDIC study. Diabetes Care. 2017;40(8):1010-6.

6. Frier BM. Hypoglycaemia in diabetes mellitus: epidemiology and clinical implications. Nat Rev Endocrinol. 2014;10(12):711-22.

7. Ostenson CG, Geelhoed-Duijvestijn P, Lahtela J, Weitgasser R, Markert Jensen M, Pedersen-Bjergaard U. Self-reported non-severe hypoglycaemic events in Europe. Diabet Med. 2014;31(1):92-101.

8. Cryer PE. Severe hypoglycemia predicts mortality in diabetes. Diabetes Care. 2012;35(9):1814-6.

9. Geddes J, Schopman JE, Zammitt NN, Frier BM. Prevalence of impaired awareness of hypoglycaemia in adults with type 1 diabetes. Diabet Med. 2008;25(4):501-4.

10. Hex N, York health economics consortium Itd UoY, York UK, Bartlett C, Wright D, et al. Estimating the current and future costs of type 1 and type 2 diabetes in the UK, including direct health costs and indirect societal and productivity costs. Diabet Med. 2017;29(7):855-62.

11. Dall TM, Mann SE, Zhang Y, Quick WW, Seifert RF, Martin J, et al. Distinguishing the Economic Costs Associated with Type 1 and Type 2 Diabetes. Popul Health Manag. 2009;12(2):103 http://wwwliebertpubcom/pop.

12. Leese GP, Wang J, Broomhall J, Kelly P, Marsden A, Morrison W, et al. Frequency of severe hypoglycemia requiring emergency treatment in type 1 and type 2 diabetes: a population-based study of health service resource use. Diabetes Care. 2003;26(4):1176-80.

13. McEwan P, Larsen Thorsted B, Wolden M, Jacobsen J, Evans M. Healthcare resource implications of hypoglycemia-related hospital admissions and inpatient hypoglycemia: retrospective record-linked cohort studies in England. BMJ Open Diabetes Res Care. 2015;3(1):e000057

14. Farmer AJ, Brockbank KJ, Keech ML, England EJ, Deakin CD. Incidence and costs of severe hypoglycaemia requiring attendance by the emergency medical services in south Central England. Diabet Med. 2012;29(11):1447-50.

15. Heller SR, Frier BM, Herslov ML, Gundgaard J, Gough SC. Severe hypoglycaemia in adults with insulin-treated diabetes: impact on healthcare resources. Diabet Med. 2016;33(4):471-7.
16. Group. DS. Training in flexible, intensive insulin management to enable dietary freedom in people with type 1 diabetes: dose adjustment for normal eating (DAFNE) randomised controlled trial. BMJ. 2002;325(7367):746

17. Pickup JC, Freeman SC, Sutton AJ. Glycaemic control in type 1 diabetes during real time continuous glucose monitoring compared with self monitoring of blood glucose: meta-analysis of randomised controlled trials using individual patient data. Bmj. 2011;343:d3805.

18. Lind M, Polonsky W, Hirsch IB, Heise T, Bolinder J, Dahlqvist S, et al. Continuous glucose monitoring vs conventional therapy for glycemic control in adults with type 1 diabetes treated with multiple daily insulin injections: the GOLD randomized clinical trial. Jama. 2017;317(4):379-87.

19. Polonsky WH, Hessler D, Ruedy KJ, Beck RW. The impact of continuous glucose monitoring on markers of quality of life in adults with type 1 diabetes: further findings from the DIAMOND randomized clinical trial. Diabetes Care. 2017;40(6):736-41.

20. Litchman ML, Allen NA. Real-time continuous glucose monitoring facilitates feelings of safety in older adults with type 1 diabetes: a qualitative study. J Diabetes Sci Technol. 2017;11(5):988-95.

21. Parkin CG, Graham C, Smolskis J. Continuous glucose monitoring use in type 1 diabetes: longitudinal analysis demonstrates meaningful improvements in $\mathrm{HbA} 1 \mathrm{c}$ and reductions in health care utilization. J Diabetes Sci Technol. 2017:11(3):522-8.

22. National Institute for Health and Care Excellence (NICE). Type 1 diabetes in adults: diagnosis and management. NICE guideline 17. Available at https:// www.nice.org.uk/guidance/ng17. Accessed 23 Oct 2019.

23. Health Innovation Network. The Integrated Hypoglycaemic Pathway. 2016. Available from: https://healthinnovationnetwork.com/projects/ theintegratedhypoglycaemicpathway/. Accessed 23 Oct 2019.

24. Sampson M, Bailey M, Clark J, Evans ML, Fong R, Hall H, et al. A new integrated care pathway for ambulance attended severe hypoglycaemia in the east of England: the eastern academic health science network (EAHSN) model. Diabetes Res Clin Pract. 2017;133:50-9.

25. Walker A, James C, Bannister M, Jobes E. Evaluation of a diabetes referral pathway for the management of hypoglycaemia following emergency contact with the ambulance service to a diabetes specialist nurse team. Emerg Med J. 2006;23(6):449-51.

26. Khunti K, Fisher H, Paul S, labal M, Davies MJ, Siriwardena AN. Severe hypoglycaemia requiring emergency medical assistance by ambulance services in the east midlands: a retrospective study. Prim Care Diabetes. 2013;7(2):159-65.

27. Field BCT, Nayar R, Kilvert A, Baxter M, Hickey J, Cummings M, et al. A retrospective observational study of people with type 1 diabetes with selfreported severe hypoglycaemia reveals high level of ambulance attendance but low levels of therapy change and specialist intervention. Diabet Med. 2018;35(9):1223.

28. International Hypoglycaemia Study Group. Glucose concentrations of less than $3.0 \mathrm{mmol} / \mathrm{l}(54 \mathrm{mg} / \mathrm{dl})$ should be reported in clinical trials: a joint position statement of the American Diabetes Association and the European Association for the Study of Diabetes. Diabetologia. 2017;60(1):3-6.

29. Heinemann L, Deiss D, Hermanns N, Graham C, Kaltheuner M, Liebl A, et al. HypoDE: research design and methods of a randomized controlled study evaluating the impact of real-time CGM usage on the frequency of CGM glucose values $<55 \mathrm{mg} / \mathrm{dl}$ in patients with type 1 diabetes and problematic hypoglycemia treated with multiple daily injections. J Diabetes Sci Technol. 2015;9(3):651-62.

30. van Beers CA, DeVries JH, Kleijer SJ, Smits MM, Geelhoed-Duijvestijn PH, Kramer $\mathrm{MH}$, et al. Continuous glucose monitoring for patients with type 1 diabetes and impaired awareness of hypoglycaemia (IN CONTROL): a randomised, open-label, crossover trial. Lancet Diabetes Endocrinol. 2016; 4(11):893-902.

31. Reddy M, Jugnee N, El Laboudi A, Spanudakis E, Anantharaja S, Oliver N. A randomized controlled pilot study of continuous glucose monitoring and flash glucose monitoring in people with type 1 diabetes and impaired awareness of hypoglycaemia. Diabet Med. 2018;35(4):483-90.

\section{Publisher's Note}

Springer Nature remains neutral with regard to jurisdictional claims in published maps and institutional affiliations. 